13.4

\title{
Замедляющая система меандрового типа на диэлектрической подложке для лампы бегущей волны миллиметрового диапазона
}

\author{
(C) P.A. Торгашов \\ Саратовский ффилиал Института радиотехники и электроники им. В.А. Котельникова РАН, Саратов, Россия \\ Саратовский национальный исследовательский государственный университет им. Н.Г. Чернышевского, Саратов, Россия \\ E-mail: torgashovra@gmail.com
}

Поступило в Редакцию 17 июля 2020 г.

В окончательной редакции 13 августа 2020 г.

Принято к публикации 18 августа 2020 г.

Предложена новая планарная замедляющая система меандрового типа на диэлектрической подложке для миниатюрной низковольтной лампы бегущей волны миллиметрового диапазона с ленточным электронным пучком большого поперечного сечения. Исследованы основные электродинамические параметры системы. Использование подобной замедляющей системы может привести к повышению коэффициента усиления и выходной мощности лампы бегущей волны усилителя.

Ключевые слова: лампа бегущей волны, меандр, замедляющая система, диэлектрическая подложка, ленточный электронный пучок.

DOI: 10.21883/PJTF.2020.23.50344.18472

Одной из ключевых задач современной вакуумной СВЧ-электроники является создание мощных и миниатюрных источников когерентного излучения миллиметрового и субмиллиметрового диапазона длин волн [1]. Применение подобных устройств возможно в современных системах высокоскоростной беспроводной передачи данных, радиолокационных системах, системах безопасности и противодействия терроризму, радиоастрономии и др.

Наибольшее внимание уделяется исследованию ламп бегущей волны (ЛБВ) О-типа, которые в короткой части миллиметрового диапазона могут обеспечить уровни мощности порядка десятков и сотен ватт при ширине полосы усиления до 30\% [1]. Ключевым элементом подобных устройств является замедляющая система (3С), в которой обеспечивается синхронизм замедленной электромагнитной волны и электронного пучка (ЭП). Однако с ростом рабочих частот поперечные размеры устройств сокращаются, а плотность тока ЭП соответственно повышается. Поэтому целесообразно использование пространственно-развитых ЗС и пучков с большим поперечным сечением, например ленточных.

Перспективными для применения в миниатюрных ЛБВ являются планарные микрополосковые ЗС на диэлектрических подложках. Подобные электродинамические структуры могут обладать высокими значениями коэффициента замедления, что в свою очередь приводит к снижению рабочих напряжений и продольных размеров устройств. Их достоинствами также являются отсутствие пролетного канала с малым диаметром, возможность использования ленточного ЭП с большим поперечным сечением, а также возможность применения современных технологий микроэлектроники (лазерная абляция, фотолитография и т.д.). Основное вни- мание уделяется 3С типа прямоугольных, $U$-образных или $V$-образных меандров на диэлектрической подложке [2-10]. В частности, в работе [7] разработана ЗС $V$-диапазона $(50-70 \mathrm{GHz})$ с рабочими напряжениями $2.5-5.5 \mathrm{kV}$. Расчеты показали, что при длине пространства взаимодействия $25 \mathrm{~mm}$ коэффициент усиления может достигать значений свыше $30 \mathrm{~dB}$.

В настоящей работе предложена новая планарная 3С, которая представляет собой два противофазных электрически связанных микрополосковых металлических меандра на диэлектрической подложке. На рис. 1 представлено схематическое изображение периода ЗС в прямоугольном волноводе. Предполагается, что в подобной системе замедленная электромагнитная волна взаимодействует с ленточным электронным пучком, который находится на расстоянии $a$ от поверхности металлического меандра.

Разработана конструкция и подобраны оптимальные размеры для 3C $V$-диапазона. Основные геометрические параметры системы представлены в таблице. Предполагается, что ЗС помещена в прямоугольный волновод стандартного сечения WR-15. Моделирование электродинамических параметров структуры проводилось с помощью программного пакета COMSOL Multiphysics [11]. Была построена модель периода 3С, на границы которого вдоль продольного направления наложены периодические граничные условия Флоке. В качестве материала полоска выбрана медь, материал подложки - кварц с диэлектрической проницаемостью $\varepsilon \approx 3.75$.

На рис. 2, а представлена дисперсионная характеристика структуры. Показаны две основные моды, для которых распределения продольной компоненты поля имеют синфазную (симметричную) и противофазную (антисимметричную) структуру. Для применения в 


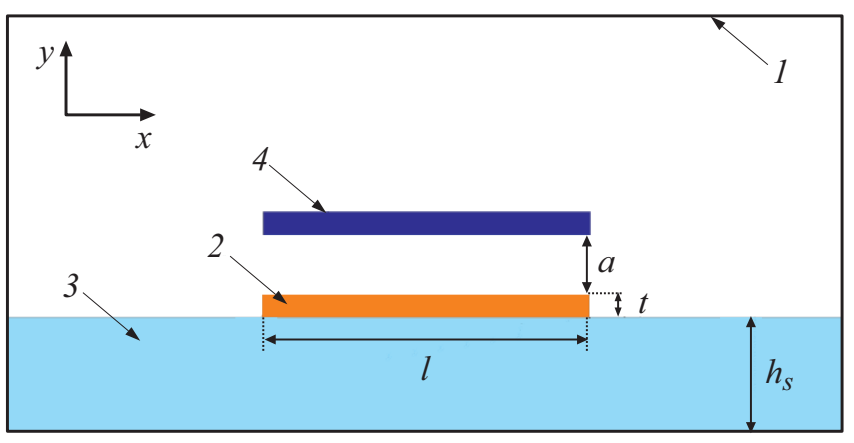

Side view

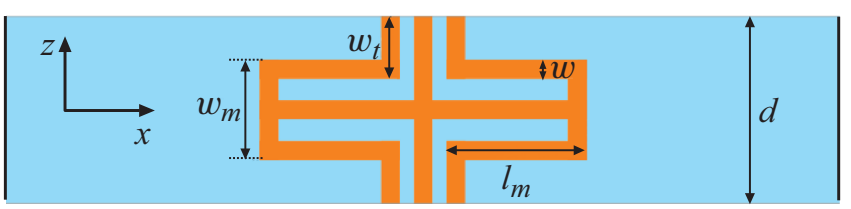

Top view

Рис. 1. Схематическое изображение периода $3 \mathrm{C}$ на диэлектрической подложке. 1 - волновод, $2-$ меандр, 3 - подложка, 4 - электронный пучок.

Геометрические параметры 3С

\begin{tabular}{l|c}
\hline \multicolumn{1}{c|}{ Геометрический параметр } & Значение \\
\hline Толщина металлического слоя $t, \mu \mathrm{m}$ & 10 \\
Период структуры $d, \mu \mathrm{m}$ & 270 \\
Ширина ЗС $l, \mu \mathrm{m}$ & 1500 \\
Ширина полоска $w, \mu \mathrm{m}$ & 30 \\
Ширина меандра $l_{m}, \mu \mathrm{m}$ & 650 \\
Ширина внешнего плеча меандра $w_{m}, \mu \mathrm{m}$ & 150 \\
Полуширина внутреннего плеча меандра $w_{t}, \mu \mathrm{m}$ & 90 \\
Толщина диэлектрической подложки $h_{s}, \mu \mathrm{m}$ & 500 \\
Размер пучка, $\mu \mathrm{m}$ & $1500 \times 100$ \\
Расстояние от проводящего слоя до пучка $a, \mu \mathrm{m}$ & 100 \\
Размер волновода, тm & $3.6 \times 1.8$
\end{tabular}

ЛБВ-усилителе необходимо использовать ту ветвь характеристики, которая обладает нормальной дисперсией. Для обеих мод нулевая пространственная гармоника обладает нормальной дисперсией. Возбуждение той или иной моды возможно с помощью соответствующей конструкции устройства ввода-вывода энергии, например с использованием копланарной линии передачи. На рис. 2, $a$ также представлена дисперсионная характеристика пучка при напряжении $5 \mathrm{kV}$. Как видно из рисунка, синхронизм возможен с обеими нормальными модами, причем частоты синхронизма оказываются близкими. Для синфазной моды коэффициент замедления принимает значения 5-8, что соответствует напряжениям в диапазоне $4-11 \mathrm{kV}$. Для противофазной моды на длинноволновом конце рабочего частотного диапазона напряжение принимает более высокие значения - до $15 \mathrm{kV}$.

На рис. 2, $b$ представлен пример распределения продольной компоненты электрического поля $E_{z}$ вдоль поперечной координаты $x$ на частоте около $65 \mathrm{GHz}$, т.е. вблизи точки синхронизма пучка с замедленной волной, на расстоянии $150 \mu \mathrm{m}$ от поверхности металлического меандра. Эти зависимости наглядно иллюстрируют симметричную и антисимметричную структуру полей. Также рис. $2, b$ показывает, что возможно использование ленточного пучка с шириной до $2000 \mu \mathrm{m}$.

Частотные зависимости сопротивления связи $R_{c}$, усредненного по поперечному сечению пучка площадью
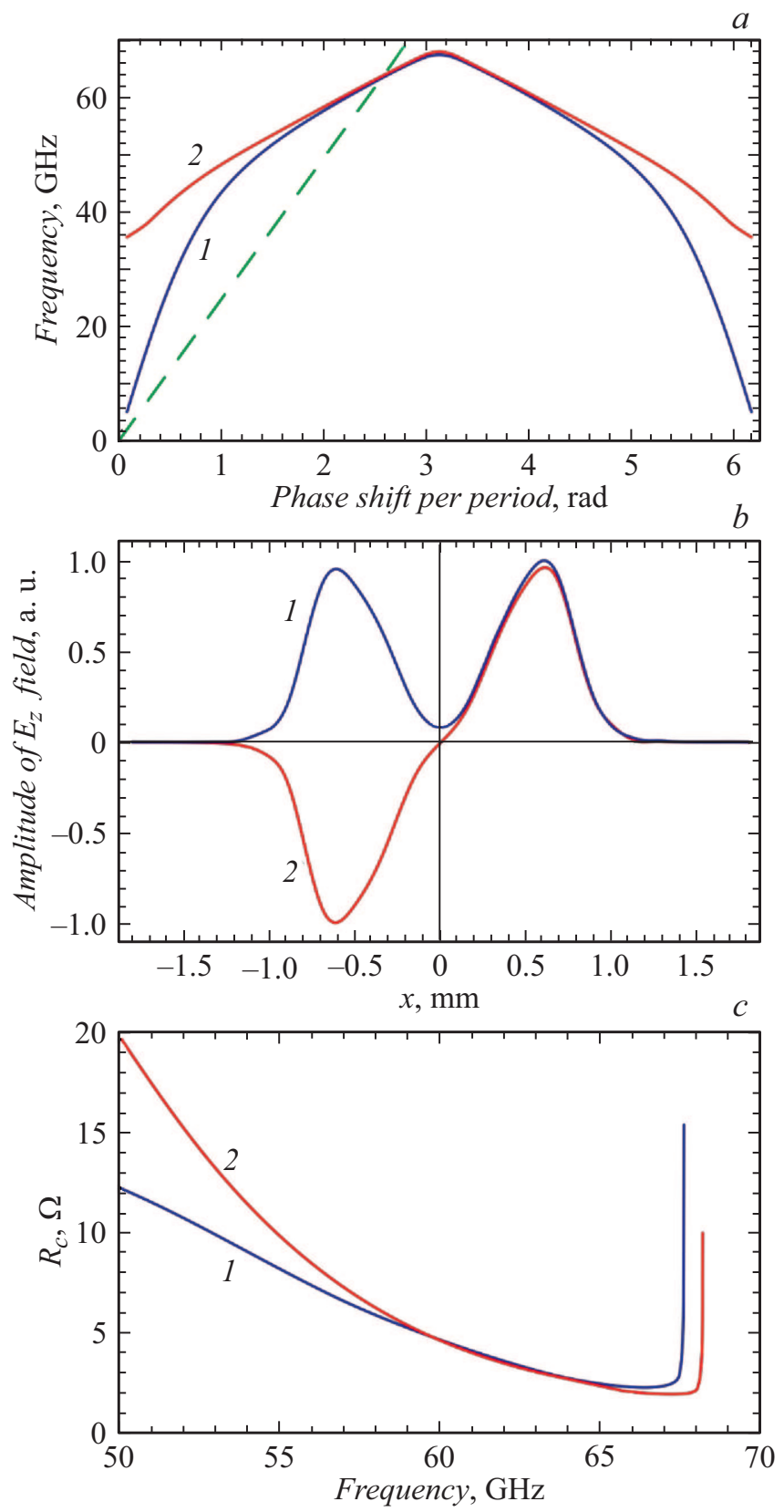

Рис. 2. Электродинамические параметры ЗС. $a-$ дисперсионная характеристика 3С (штриховой линией показана дисперсионная характеристика пучка при напряжении $5 \mathrm{kV}$ ); $b-$ распределения продольной компоненты электрического поля $E_{z}$ вдоль поперечной координаты $x ; c-$ зависимость сопротивления связи $R_{c}$ от частоты. 1 - синфазная мода, 2 - противофазная мода. 
$1500 \times 100 \mu \mathrm{m}$, представлены на рис. 2,c. Расстояние от нижнего края пучка до металлической поверхности $100 \mu \mathrm{m}$. Хотя при удалении пучка от поверхности меандра сопротивление связи резко убывает, что связано с экспоненциальным убыванием продольной компоненты электрического поля [5,7], приближение пучка к ЗС на меньшие расстояния нецелесообразно ввиду возможного оседания электронов на диэлектрик. Выбранные значения толщины пучка и расстояния до поверхности меандра являются типичными для приборов миллиметрового диапазона с планарными ЗС на диэлектрических подложках [3-10]. Как видно из рис. 2,c, при выбранных параметрах $R_{c}$ принимает довольно высокие значения, причем на низкочастотном краю рабочего диапазона для противофазной моды оно достигает $20 \Omega$. С ростом частоты $R_{c}$ уменьшается до примерно $2 \Omega$, однако при приближении к границе полосы пропускания начинает расти, стремясь к бесконечности.

Основным преимуществом предложенной 3С является возможность использования широкого ленточного ЭП, что позволяет увеличить ток и, следовательно, повысить выходную мощность. Например, для ЛБВ с 3С в виде одиночного меандра, представленной в [7], при токе пучка $100 \mathrm{~mA}$ и поперечных размерах пучка $650 \times 100 \mu \mathrm{m}$ плотность тока составляет примерно $154 \mathrm{~A} / \mathrm{cm}^{2}$. Для системы, рассматриваемой в настоящей работе, ширина пучка составляла $1500 \mu \mathrm{m}$, т. е. ток пучка той же плотности будет составлять примерно $230 \mathrm{~mA}$, т. е. увеличится в 2.3 раза.

На рис. 3 представлены результаты расчетов режимов усиления ЛБВ для обоих типов 3С, которые были проведены на основе уравнений одномерной нелинейной теории ЛБВ (см., например, [8]). Напряжение пучка составляло $5 \mathrm{kV}$. При расчете режимов усиления учитывались омические потери, которые в соответствии с полученными ранее результатами для одиночного меандра были выбраны равными $0.6 \mathrm{~dB} / \mathrm{mm}$. Длина ЗС составляла $25 \mathrm{~mm}$, что соответствует 93 периодам предлагаемой 3С и 125 периодам одиночного меандра.

На рис. 3,a представлены частотные зависимости коэффициента усиления в режиме малого входного сигнала. Как видно из рис. $3, a$, максимальные значения коэффициента усиления для обеих систем примерно одинаковы и достигают значений 25-27 dB. Вдали от полосы усиливаемых частот коэффициент усиления стремится к значению $-15 \mathrm{~dB}$, что соответствует выбранному значению холодного ослабления.

Рис. $3, b$ иллюстрирует нелинейные характеристики ЛБВ-усилителя. Представлены зависимости выходной мощности $P_{\text {out }}$ от мощности входного сигнала $P_{\text {in }}$ на тех частотах, на которых выходная мощность достигает максимума. Видно, что максимальная выходная мощность $P_{\text {out }}$ ЛБВ с предлагаемой ЗС превышает выходную мощность ЛБВ с одиночным меандром более чем в 2 раза.
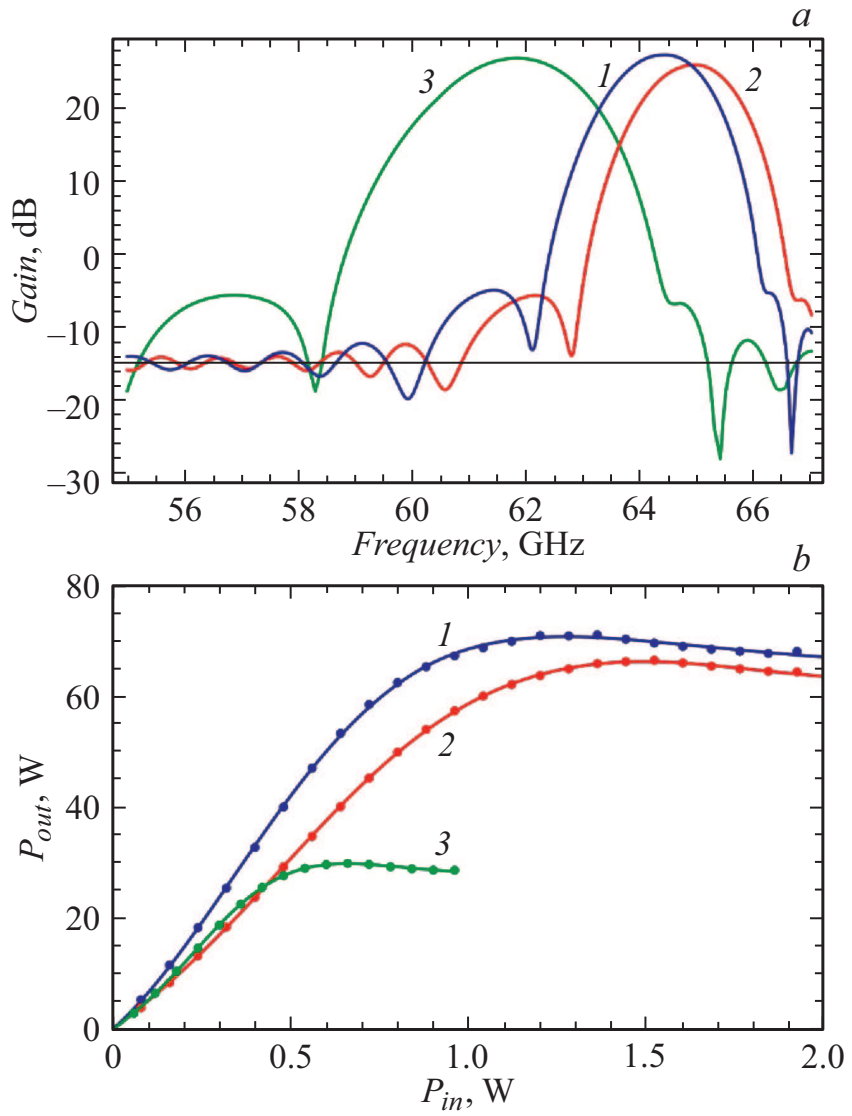

Рис. 3. Выходные характеристики ЛБВ при плотности тока $154 \mathrm{~A} / \mathrm{cm}^{2}$, напряжении $5 \mathrm{kV}$ и длине системы $25 \mathrm{~mm} . a-$ зависимость коэффициента усиления в режиме малого входного сигнала от частоты: 1 - синфазная мода, 2 - противофазная мода, $3-3$ в в виде одиночного меандра; $b-$ зависимость выходной мощности $P_{\text {out }}$ от входной $P_{\text {in }}: 1-$ синфазная мода на частоте входного сигнала $65.5 \mathrm{GHz}, 2$ - противофазная мода на частоте входного сигнала $66 \mathrm{GHz}, 3$ - основная мода ЗС в виде одиночного меандра на частоте входного сигнала $63.5 \mathrm{GHz}$.

В настоящей работе представлена конструкция новой ЗС меандрового типа для миниатюрного низковольтного ЛБВ-усилителя миллиметрового диапазона. Исследованы основные электродинамические параметры 3C $V$-диапазона и показано, что для подобной ЗС характерны как высокие значения сопротивления связи $(2-20 \Omega)$, так и относительно низкие значения рабочих напряжений $(4-15 \mathrm{kV})$. Преимуществом представленной ЗС является возможность использования широкого ЭП, что позволяет существенно повысить выходную мощность ЛБВ в режиме насыщения. Дальнейшие исследования будут направлены на разработку конструкции ввода/вывода энергии для эффективного возбуждения той или иной моды, а также на более детальное изучение выходных характеристик прибора с целью их оптимизации. Интерес также представляет возможность использования двухлучевого ЭП, что может снизить требования к магнитной фокусирующей системе. 


\section{Благодарности}

Автор выражает благодарность Н.М. Рыскину за ценные научные советы.

\section{Финансирование работы}

Работа выполнена в рамках государственного задания Института радиотехники и электроники им. В.А. Котельникова РАН.

\section{Конфликт интересов}

Автор заявляет, что у него нет конфликта интересов.

\section{Список литературы}

[1] Dhillon S.S., Vitiello M.S., Linfield E.H., Davies A.G., Hoffmann M.C., Booske J., Paoloni C., Gensch M., Weightman P., Williams G.P., Castro-Camus E., Cumming D.R.S., Simoens F., Escorcia-Carranza I., Grant J., Lucyszyn S., Kuwata-Gonokami M., Konishi K., Koch M., Schmuttenmaer C.A., Cocker T.L., Huber R., Markelz A.G., Taylor Z.D., Wallace V.P., Zeitler J.A., Sibik J., Korter T.M., Ellison B., Rea S., Goldsmith P., Cooper K.B., Appleby R., Pardo D., Huggard P.G., Krozer V., Shams H., Fice M., Renaud C., Seeds A., Stöhr A., Naftaly M., Ridler N., Clarke R., Cunningham J.E., Johnston M.B. // J. Phys. D: Appl. Phys. 2017. V. 50. N 4. P. 043001.

DOI: 10.1088/1361-6463/50/4/043001

[2] Sumathy M., Augustin D., Datta S.K., Christie L., Kumar L. // IEEE Trans. Electron Dev. 2013. V. 60. N 5. P. 1769-1775. DOI: 10.1109/TED.2013.2252179

[3] Ulisse G., Krozer V. // IEEE Electron Dev. Lett. 2017. V. 38. N 1. P. 126-129. DOI: 10.1109/LED.2016.2627602

[4] Bai N., Feng C., Liu Y., Fan H., Shen C., Sun X. // IEEE Trans. Electron Dev. 2017. V. 64. N 7. P. 2949-2954. DOI: 10.1109/TED.2017.2706368

[5] Ding C., Wei Y., Li Q., Zhang L., Guo G., Gong Y. // J. Electromagn. Waves Appl. 2017. V. 31. N 17. P. 1938 -1946. DOI: 10.1080/09205071.2017.1358109

[6] Galdetskiy A., Rakova E. // Proc. 18th IEEE Int. Vac. Electron. Conf. (IVEC). London, 2017. P. 1-2.

DOI: 10.1109/IVEC.2017.8289680

[7] Ryskin N.M., Rozhnev A.G., Starodubov A.V., Serdobintsev A.A., Pavlov A.M., Benedik A.I., Torgashov R.A., Torgashov G.V., Sinitsyn N.I. // IEEE Electron Dev. Lett. 2018. V. 39. N 5. P. 757-760. DOI: 10.1109/LED.2018.2821770

[8] Торгашов Р.А., Рыскин Н.М., Рожнев А.Г., Стародубов А.В., Сердобинщев А.А., Павлов А.М., Галушка В.В., Бахтеев И.Ш., Молчанов С.Ю. // ЖТФ. 2020. Т. 90. В. 4. C. 686-692. DOI: 10.21883/JTF.2020.04.49096.294-19 [Пер. версия: $10.1134 / \mathrm{S} 1063784220040222]$.

[9] Wang S., Aditya S., Xia X., Ali Z., Miao J. // IEEE Trans. Electron Dev. 2018. V. 65. N 6. P. 2142-2148. DOI: $10.1109 /$ TED.2018.2798575

[10] Wang S., Aditya S., Xia X., Ali Z., Miao J., Zheng Y. // IEEE Trans. Plasma Sci. 2019. V. 47. N 10. P. 4650-4657. DOI: $10.1109 /$ TPS.2019.2940254
[11] Comsol Multiphysics Engineering Simulation Software (COMSOL Inc., Burlington, MA, USA) [Электронный ресурс]. Режим доступа:

https://www.comsol.com/comsol-multiphysics 